\title{
The q-Onsager Algebra and the Universal Askey-Wilson Algebra
}

\author{
Paul TERWILLIGER
}

Department of Mathematics, University of Wisconsin, Madison, WI 53706-1388, USA

E-mail: terwilli@math.wisc.edu

Received January 25, 2018, in final form May 01, 2018; Published online May 07, 2018

https://doi.org/10.3842/SIGMA.2018.044

\begin{abstract}
Recently Pascal Baseilhac and Stefan Kolb obtained a PBW basis for the $q$ Onsager algebra $\mathcal{O}_{q}$. They defined the PBW basis elements recursively, and it is obscure how to express them in closed form. To mitigate the difficulty, we bring in the universal Askey-Wilson algebra $\Delta_{q}$. There is a natural algebra homomorphism $\sharp$ : $\mathcal{O}_{q} \rightarrow \Delta_{q}$. We apply $\downarrow$ to the above PBW basis, and express the images in closed form. Our results make heavy use of the Chebyshev polynomials of the second kind.
\end{abstract}

Key words: q-Onsager algebra; universal Askey-Wilson algebra; Chebyshev polynomial

2010 Mathematics Subject Classification: 33D80; 17B40

\section{Introduction}

In the 1944 paper [25] Lars Onsager obtained the free energy of the two-dimensional Ising model in a zero magnetic field. In that paper an infinite-dimensional Lie algebra was introduced; this algebra is now called the Onsager algebra and denoted by $\mathcal{O}$. Onsager defined his algebra by giving a linear basis and the action of the Lie bracket on the basis. In [26] Perk gave a presentation of $\mathcal{O}$ by generators and relations. This presentation involves two generators and two relations, called the Dolan/Grady relations [17]. This presentation is discussed in [30, Remark 9.1]. Via this presentation, the universal enveloping algebra of $\mathcal{O}$ admits a $q$-deformation $\mathcal{O}_{q}$ called the $q$-Onsager algebra $[4,29]$. The algebra $\mathcal{O}_{q}$ is associative and infinite-dimensional. It is defined by two generators and two relations called the $q$-Dolan/Grady relations; these are given in (2.2), (2.3) below. The $q$-Dolan/Grady relations first appeared in algebraic combinatorics, in the study of $Q$-polynomial distance-regular graphs [27, Lemma 5.4]. Shortly thereafter they appeared in physics, in the study of statistical mechanical models [4, Section 2]. Up to the present, the representation theory of $\mathcal{O}_{q}$ remains an active area of research in mathematics $[19,21,22,28,29,30,31,32,33]$ and physics $[3,4,5,6,7,8,9,10,11,12,14,15]$. This theory involves a linear algebraic object called a tridiagonal pair [20]. A finite-dimensional irreducible $\mathcal{O}_{q}$-module is essentially the same thing as a tridiagonal pair of $q$-Racah type [29, Theorem 3.10]. These tridiagonal pairs are classified up to isomorphism in [21, Theorem 3.3]. In [22, Theorem 2.1], Ito and the present author gave a linear basis for $\mathcal{O}_{q}$, called the zigzag basis. More information about this basis can be found in [32, Note 4.7]. In [7], Baseilhac and Belliard conjectured another linear basis for $\mathcal{O}_{q}$; this one is motivated by how $\mathcal{O}_{q}$ is related to the reflection equation algebra [11, 14]. In [13], Baseilhac and Kolb introduced two automorphisms $T_{0}, T_{1}$ of $\mathcal{O}_{q}$ that are roughly analogous to the Lusztig automorphisms of $U_{q}\left(\widehat{\mathfrak{s l}}_{2}\right)$. They used $T_{0}, T_{1}$ and a method of Damiani [16] to obtain a Poincaré-Birkhoff-Witt (or PBW) basis for $\mathcal{O}_{q}[13$, Theorem 4.3]. In our view this PBW basis is important and worthy of further

This paper is a contribution to the Special Issue on Orthogonal Polynomials, Special Functions and Applications (OPSFA14). The full collection is available at https://www.emis.de/journals/SIGMA/OPSFA2017.html 
study. In the present paper we study the following aspect. In [13, Section 3.1] the PBW basis is defined recursively, and it is obscure how to express it in closed form. In order to mitigate the difficulty, we bring in a related algebra which we now describe. In [34] Zhedanov introduced the Askey-Wilson algebra AW(3) and used it to describe the Askey-Wilson polynomials. In [31] the present author introduced a central extension of AW(3), called the universal Askey-Wilson algebra $\Delta_{q}$. In [18], Hau-Wen Huang classified up to isomorphism the finite-dimensional irreducible $\Delta_{q}$-modules for $q$ not a root of unity. A linear basis for $\Delta_{q}$ is given in [31, Theorem 7.5]. There is a natural algebra homomorphism $\sharp: \mathcal{O}_{q} \rightarrow \Delta_{q}$ [31, Definition 10.4]; this is described below (2.23) in the present paper. We use $\measuredangle$ to describe the PBW basis for $\mathcal{O}_{q}$ in the following way. We apply $\downarrow$ to the PBW basis vectors and consider their images in $\Delta_{q}$. We express these images explicitly in the linear basis for $\Delta_{q}$ mentioned above. Our main results are Theorems 5.5, 5.6. These results make heavy use of the Chebyshev polynomials of the second kind [23, 24].

\section{Preliminaries}

We now begin our formal argument. Recall the natural numbers $\mathbb{N}=\{0,1,2, \ldots\}$ and integers $\mathbb{Z}=\{0, \pm 1, \pm 2, \ldots\}$. Let $\mathbb{F}$ denote an algebraically closed field with characteristic zero. All the algebras discussed in this paper are over $\mathbb{F}$; those without the Lie prefix are associative and have a multiplicative identity. Fix a nonzero $q \in \mathbb{F}$ that is not a root of 1 . Recall the notation

$$
[n]_{q}=\frac{q^{n}-q^{-n}}{q-q^{-1}}, \quad n \in \mathbb{Z}
$$

We will be discussing the $q$-Onsager algebra $\mathcal{O}_{q}$ and the universal Askey-Wilson algebra $\Delta_{q}$. We now recall these algebras.

The algebra $\mathcal{O}_{q}$ (see [4, Section 2], [29, Definition 3.9]) is defined by generators $A, B$ and relations

$$
\begin{aligned}
& A^{3} B-[3]_{q} A^{2} B A+[3]_{q} A B A^{2}-B A^{3}=\left(q^{2}-q^{-2}\right)^{2}(B A-A B), \\
& B^{3} A-[3]_{q} B^{2} A B+[3]_{q} B A B^{2}-A B^{3}=\left(q^{2}-q^{-2}\right)^{2}(A B-B A) .
\end{aligned}
$$

The relations (2.2), (2.3) are called the $q$-Dolan/Grady relations. In [13], Baseilhac and Kolb introduced the automorphisms $T_{0}, T_{1}$ of $\mathcal{O}_{q}$. These automorphisms satisfy

$$
\begin{aligned}
& T_{0}(A)=A, \quad T_{0}(B)=B+\frac{q A^{2} B-\left(q+q^{-1}\right) A B A+q^{-1} B A^{2}}{\left(q-q^{-1}\right)\left(q^{2}-q^{-2}\right)}, \\
& T_{1}(B)=B, \quad T_{1}(A)=A+\frac{q B^{2} A-\left(q+q^{-1}\right) B A B+q^{-1} A B^{2}}{\left(q-q^{-1}\right)\left(q^{2}-q^{-2}\right)} .
\end{aligned}
$$

The inverse automorphisms satisfy

$$
\begin{array}{ll}
T_{0}^{-1}(A)=A, & T_{0}^{-1}(B)=B+\frac{q^{-1} A^{2} B-\left(q+q^{-1}\right) A B A+q B A^{2}}{\left(q-q^{-1}\right)\left(q^{2}-q^{-2}\right)}, \\
T_{1}^{-1}(B)=B, & T_{1}^{-1}(A)=A+\frac{q^{-1} B^{2} A-\left(q+q^{-1}\right) B A B+q A B^{2}}{\left(q-q^{-1}\right)\left(q^{2}-q^{-2}\right)} .
\end{array}
$$

In [13], Baseilhac and Kolb used $T_{0}$ and $T_{1}$ to define some elements in $\mathcal{O}_{q}$, denoted

$$
\left\{B_{n \delta+\alpha_{0}}\right\}_{n=0}^{\infty}, \quad\left\{B_{n \delta+\alpha_{1}}\right\}_{n=0}^{\infty}, \quad\left\{B_{n \delta}\right\}_{n=1}^{\infty} .
$$

The elements (2.8) were shown to be a PBW basis for $\mathcal{O}_{q}$, provided that $q$ is transcendental over $\mathbb{F}[13$, Theorem 4.3]. By definition 


\begin{tabular}{c|cc}
$n$ & $B_{n \delta+\alpha_{0}}$ & $B_{n \delta+\alpha_{1}}$ \\
\hline 0 & $A$ & $B$ \\
1 & $T_{0}(B)$ & $T_{1}^{-1}(A)$ \\
2 & $T_{0} T_{1}(A)$ & $T_{1}^{-1} T_{0}^{-1}(B)$ \\
3 & $T_{0} T_{1} T_{0}(B)$ & $T_{1}^{-1} T_{0}^{-1} T_{1}^{-1}(A)$ \\
4 & $T_{0} T_{1} T_{0} T_{1}(A)$ & $T_{1}^{-1} T_{0}^{-1} T_{1}^{-1} T_{0}^{-1}(B)$ \\
$\vdots$ & $\vdots$ & $\vdots$
\end{tabular}

and for $n \geq 1$,

$$
B_{n \delta}=q^{-2} B_{(n-1) \delta+\alpha_{1}} A-A B_{(n-1) \delta+\alpha_{1}}+\left(q^{-2}-1\right) \sum_{\ell=0}^{n-2} B_{\ell \delta+\alpha_{1}} B_{(n-\ell-2) \delta+\alpha_{1}} .
$$

By [13, Proposition 5.12] the elements $\left\{B_{n \delta}\right\}_{n=1}^{\infty}$ mutually commute. We have $B_{\delta}=q^{-2} B A-A B$. Define $\tilde{B}_{\delta}=q^{-2} A B-B A$. By [13, Lemma 3.1] we have $T_{1}\left(B_{\delta}\right)=\tilde{B}_{\delta}$ and $T_{0}\left(\tilde{B}_{\delta}\right)=B_{\delta}$. So as noted in [13, Lemma 3.1],

$$
T_{0} T_{1}\left(B_{\delta}\right)=B_{\delta}, \quad T_{1}^{-1} T_{0}^{-1}\left(B_{\delta}\right)=B_{\delta} .
$$

Next we recall the universal Askey-Wilson algebra $\Delta_{q}$ [31, Definition 1.2]. This algebra is defined by generators and relations. The generators are $A, B, C$. The relations assert that each of the following is central in $\Delta_{q}$ :

$$
A+\frac{q B C-q^{-1} C B}{q^{2}-q^{-2}}, \quad B+\frac{q C A-q^{-1} A C}{q^{2}-q^{-2}}, \quad C+\frac{q A B-q^{-1} B A}{q^{2}-q^{-2}} .
$$

For the above three central elements, multiply each by $q+q^{-1}$ to get $\alpha, \beta, \gamma$. Thus

$$
\begin{aligned}
& A+\frac{q B C-q^{-1} C B}{q^{2}-q^{-2}}=\frac{\alpha}{q+q^{-1}}, \\
& B+\frac{q C A-q^{-1} A C}{q^{2}-q^{-2}}=\frac{\beta}{q+q^{-1}}, \\
& C+\frac{q A B-q^{-1} B A}{q^{2}-q^{-2}}=\frac{\gamma}{q+q^{-1}} .
\end{aligned}
$$

Each of $\alpha, \beta, \gamma$ is central in $\Delta_{q}$. By [31, Corollary 8.3] the center $Z\left(\Delta_{q}\right)$ is generated by $\alpha, \beta$, $\gamma, \Omega$ where

$$
\Omega=q A B C+q^{2} A^{2}+q^{-2} B^{2}+q^{2} C^{2}-q A \alpha-q^{-1} B \beta-q C \gamma .
$$

The element $\Omega$ is called the Casimir element. By [31, Theorem 8.2] the following is a linear basis for the $\mathbb{F}$-vector space $Z\left(\Delta_{q}\right)$ :

$$
\Omega^{\ell} \alpha^{r} \beta^{s} \gamma^{t}, \quad \ell, r, s, t \geq 0 .
$$

We mention two bases for $\Delta_{q}$. By [31, Theorem 4.1], the following is a linear basis for the $\mathbb{F}$-vector space $\Delta_{q}$ :

$$
A^{i} B^{j} C^{k} \alpha^{r} \beta^{s} \gamma^{t}, \quad i, j, k, r, s, t \geq 0 .
$$

By [31, Theorem 7.5], the following is a linear basis for the $\mathbb{F}$-vector space $\Delta_{q}$ :

$$
A^{i} B^{j} C^{k} \Omega^{\ell} \alpha^{r} \beta^{s} \gamma^{t}, \quad i, j, k, \ell, r, s, t \geq 0, \quad i j k=0 .
$$

For convenience we will work with the basis (2.16). 
Shortly we will discuss how $\Delta_{q}$ is related to $\mathcal{O}_{q}$. To aid in this discussion we recall from [31, Section 2] a second presentation of $\Delta_{q}$. By (2.11)-(2.13) the algebra $\Delta_{q}$ is generated by $A, B, \gamma$. Moreover

$$
\begin{aligned}
& C=\frac{\gamma}{q+q^{-1}}-\frac{q A B-q^{-1} B A}{q^{2}-q^{-2}}, \\
& \alpha=\frac{B^{2} A-\left(q^{2}+q^{-2}\right) B A B+A B^{2}+\left(q^{2}-q^{-2}\right)^{2} A+\left(q-q^{-1}\right)^{2} B \gamma}{\left(q-q^{-1}\right)\left(q^{2}-q^{-2}\right)}, \\
& \beta=\frac{A^{2} B-\left(q^{2}+q^{-2}\right) A B A+B A^{2}+\left(q^{2}-q^{-2}\right)^{2} B+\left(q-q^{-1}\right)^{2} A \gamma}{\left(q-q^{-1}\right)\left(q^{2}-q^{-2}\right)} .
\end{aligned}
$$

By [31, Theorem 2.2] the algebra $\Delta_{q}$ has a presentation by generators $A, B, \gamma$ and relations

$$
\begin{aligned}
& A^{3} B-[3]_{q} A^{2} B A+[3]_{q} A B A^{2}-B A^{3}=\left(q^{2}-q^{-2}\right)^{2}(B A-A B), \\
& B^{3} A-[3]_{q} B^{2} A B+[3]_{q} B A B^{2}-A B^{3}=\left(q^{2}-q^{-2}\right)^{2}(A B-B A), \\
& A^{2} B^{2}-B^{2} A^{2}+\left(q^{2}+q^{-2}\right)(B A B A-A B A B)=\left(q-q^{-1}\right)^{2}(B A-A B) \gamma, \\
& \gamma A=A \gamma, \quad \gamma B=B \gamma .
\end{aligned}
$$

The relations (2.20), (2.21) are the $q$-Dolan/Grady relations. Consequently there exists an algebra homomorphism $\sharp$ : $\mathcal{O}_{q} \rightarrow \Delta_{q}$ that sends $A \mapsto A$ and $B \mapsto B$. This homomorphism is not injective by [31, Theorem 10.9].

In order to clarify the nature of $T_{0}, T_{1}$, t we now introduce some automorphisms $t_{0}, t_{1}$ of $\Delta_{q}$ such that $t_{0} \curvearrowleft=\curvearrowleft T_{0}$ and $t_{1} \curvearrowleft=\left\llcorner T_{1}\right.$. To this end, we recall from [31, Section 3] how the modular group $\mathrm{PSL}_{2}(\mathbb{Z})$ acts on $\Delta_{q}$ as a group of automorphisms. By [1] the group $\mathrm{PSL}_{2}(\mathbb{Z})$ has a presentation by generators $\rho, \sigma$ and relations $\rho^{3}=1, \sigma^{2}=1$. Earlier in this section we gave two presentations of $\Delta_{q}$. Using these presentations we find that $\mathrm{PSL}_{2}(\mathbb{Z})$ acts on $\Delta_{q}$ as a group of automorphisms in the following way:

\begin{tabular}{c|ccc|ccc}
$u$ & $A$ & $B$ & $C$ & $\alpha$ & $\beta$ & $\gamma$ \\
\hline$\rho(u)$ & $B$ & $C$ & $A$ & $\beta$ & $\gamma$ & $\alpha$ \\
$\sigma(u)$ & $B$ & $A$ & $C+\frac{A B-B A}{q-q^{-1}}$ & $\beta$ & $\alpha$ & $\gamma$
\end{tabular}

This action is faithful by [31, Theorem 3.13]. From the table $(2.24)$ we see that the $\mathrm{PSL}_{2}(\mathbb{Z})$ generators $\rho, \sigma$ each permute $\alpha, \beta, \gamma$. This gives a group homomorphism from $\mathrm{PSL}_{2}(\mathbb{Z})$ onto the symmetric group $S_{3}$. Let $\mathbb{P}$ denote the kernel of the homomorphism. Thus $\mathbb{P}$ is a normal subgroup of $\mathrm{PSL}_{2}(\mathbb{Z})$, and the quotient group $\operatorname{PSL}_{2}(\mathbb{Z}) / \mathbb{P}$ is isomorphic to $S_{3}$. The cosets of $\mathbb{P}$ in $\mathrm{PSL}_{2}(\mathbb{Z})$ are

$$
\mathbb{P}, \quad \rho \mathbb{P}, \quad \rho^{2} \mathbb{P}, \quad \sigma \mathbb{P}, \quad \rho \sigma \mathbb{P}, \quad \rho^{2} \sigma \mathbb{P} .
$$

We remark that in the literature the groups $\mathrm{PSL}_{2}(\mathbb{Z})$ and $\mathbb{P}$ are often denoted by $\Gamma$ and $\Gamma(2)$, respectively; see for example [1, 2]. Define

$$
t_{0}=\left(\rho^{2} \sigma\right)^{2}=(\sigma \rho)^{-2}, \quad t_{1}=\left(\sigma \rho^{2}\right)^{2}=(\rho \sigma)^{-2} .
$$

Using (2.24), (2.25) we obtain $t_{0}, t_{1} \in \mathbb{P}$. By [2, Proposition 4] the group $\mathbb{P}$ is freely generated by $t_{0}^{ \pm 1}, t_{1}^{ \pm 1}$. Using $(2.17),(2.24),(2.25)$ we obtain

$$
\begin{aligned}
& t_{0}(A)=A, \quad t_{0}(B)=B+\frac{q A^{2} B-\left(q+q^{-1}\right) A B A+q^{-1} B A^{2}}{\left(q-q^{-1}\right)\left(q^{2}-q^{-2}\right)}, \\
& t_{1}(B)=B, \quad t_{1}(A)=A+\frac{q B^{2} A-\left(q+q^{-1}\right) B A B+q^{-1} A B^{2}}{\left(q-q^{-1}\right)\left(q^{2}-q^{-2}\right)}
\end{aligned}
$$


and

$$
\begin{array}{ll}
t_{0}^{-1}(A)=A, & t_{0}^{-1}(B)=B+\frac{q^{-1} A^{2} B-\left(q+q^{-1}\right) A B A+q B A^{2}}{\left(q-q^{-1}\right)\left(q^{2}-q^{-2}\right)}, \\
t_{1}^{-1}(B)=B, & t_{1}^{-1}(A)=A+\frac{q^{-1} B^{2} A-\left(q+q^{-1}\right) B A B+q A B^{2}}{\left(q-q^{-1}\right)\left(q^{2}-q^{-2}\right)} .
\end{array}
$$

The actions (2.26)-(2.29) match (2.4)-(2.7). Consequently the following diagrams commute:

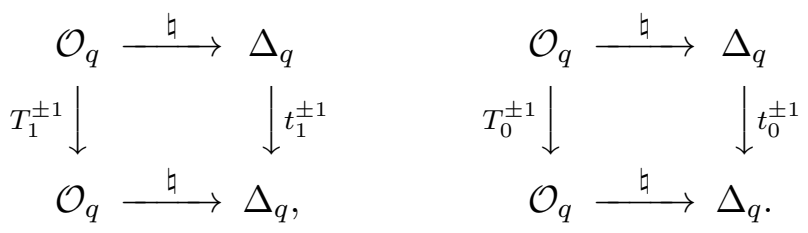

Let $\operatorname{Aut}\left(\mathcal{O}_{q}\right)$ denote the automorphism group of $\mathcal{O}_{q}$. Let $G$ denote the subgroup of $\operatorname{Aut}\left(\mathcal{O}_{q}\right)$ generated by $T_{0}^{ \pm 1}, T_{1}^{ \pm 1}$. Since $\mathbb{P}$ is freely generated by $t_{0}^{ \pm 1}, t_{1}^{ \pm 1}$ there exists a group homomorphism $\varepsilon: \mathbb{P} \rightarrow G$ that sends $t_{0}^{ \pm 1} \mapsto T_{0}^{ \pm 1}$ and $t_{1}^{ \pm 1} \mapsto T_{1}^{ \pm 1}$. Using the commuting diagrams (2.30) one finds that for $\pi \in \mathbb{P}$ the following diagram commutes:

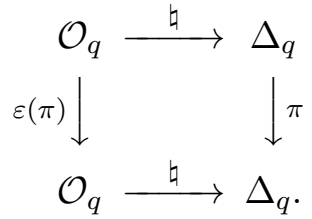

We now prove that $\varepsilon$ is an isomorphism. By construction $\varepsilon$ is surjective. We show that $\varepsilon$ is injective. Given an element $r$ in the kernel of $\varepsilon$, we show that $r$ is the identity in $\mathbb{P}$. To this end, we show that $r$ fixes the generators $A, B, \gamma$ of $\Delta_{q}$. The map $\varepsilon(r)$ is the identity in $G$, so $\varepsilon(r)$ fixes the elements $A, B$ of $\mathcal{O}_{q}$. By the commuting diagram (2.31) the map $r$ fixes the elements $A, B$ of $\Delta_{q}$. Also $r$ fixes $\gamma$ since $r \in \mathbb{P}$ and everything in $\mathbb{P}$ fixes $\gamma$. We have shown that $r$ fixes the generators $A, B, \gamma$ of $\Delta_{q}$ so $r$ is the identity in $\mathbb{P}$. Consequently $\varepsilon$ is injective and hence an isomorphism.

It is mentioned in [13, Section 2.3] that one expects $G$ to be freely generated by $T_{0}^{ \pm 1}, T_{1}^{ \pm 1}$. This is now easily proven as follows. The group $\mathbb{P}$ is freely generated by $t_{0}^{ \pm 1}, t_{1}^{ \pm 1}$. Applying the isomorphism $\varepsilon: \mathbb{P} \rightarrow G$ we find that $G$ is freely generated by $T_{0}^{ \pm 1}, T_{1}^{ \pm 1}$.

Next we consider how the map $\downarrow: \mathcal{O}_{q} \rightarrow \Delta_{q}$ acts on the elements (2.8). For these elements we retain the same notation for their images under $\downarrow$. Our goal is to obtain these images in closed form, in terms of the basis (2.16). In order to obtain these images, it is convenient to bring in the Chebyshev polynomials of the second kind. These polynomials are reviewed in the next section.

\section{The Chebyshev polynomials}

In this section we review the Chebyshev polynomials of the second kind; see [23, 24] for further details. Let $x$ denote an indeterminate. Let $\mathbb{F}[x]$ denote the $\mathbb{F}$-algebra consisting of the polynomials in $x$ that have all coefficients in $\mathbb{F}$.

Definition 3.1 (see $\left[24\right.$, p. 4]). For $n \in \mathbb{N}$ define $U_{n} \in \mathbb{F}[x]$ by

$$
U_{0}=1, \quad U_{1}=x, \quad x U_{n}=U_{n+1}+U_{n-1}, \quad n \geq 1 .
$$

The polynomial $U_{n}$ is monic and degree $n$. We call $U_{n}$ the $n$th Chebyshev polynomial of the second kind. For notational convenience define $U_{n}=0$ for all integers $n<0$. 
Note 3.2. The above polynomials $U_{n}$ are normalized to be monic. This normalization differs from the one in [23, Section 9.8.2]. To go from our normalization to the one in [23, Section 9.8.2], replace $x$ by $2 x$.

In the table below we display $U_{n}$ for $0 \leq n \leq 9$.

\begin{tabular}{c|c}
$n$ & $U_{n}$ \\
\hline 0 & 1 \\
1 & $x$ \\
2 & $x^{2}-1$ \\
3 & $x^{3}-2 x$ \\
4 & $x^{4}-3 x^{2}+1$ \\
5 & $x^{5}-4 x^{3}+3 x$ \\
6 & $x^{6}-5 x^{4}+6 x^{2}-1$ \\
7 & $x^{7}-6 x^{5}+10 x^{3}-4 x$ \\
8 & $x^{8}-7 x^{6}+15 x^{4}-10 x^{2}+1$ \\
9 & $x^{9}-8 x^{7}+21 x^{5}-20 x^{3}+5 x$
\end{tabular}

By [24, pp. 332-333],

$$
U_{n}(x)=\sum_{i=0}^{\lfloor n / 2\rfloor}(-1)^{i}\left(\begin{array}{c}
n-i \\
i
\end{array}\right) x^{n-2 i}, \quad n \in \mathbb{N} .
$$

Next we express the polynomials $U_{n}$ in a more closed form. Let $z$ denote an indeterminate. Let $\mathbb{F}\left[z, z^{-1}\right]$ denote the $\mathbb{F}$-algebra consisting of the Laurent polynomials in $z$ that have all coefficients in $\mathbb{F}$. This algebra has an automorphism that sends $z \mapsto z^{-1}$. An element of $\mathbb{F}\left[z, z^{-1}\right]$ that is fixed by the automorphism is called symmetric. The symmetric elements form a subalgebra of $\mathbb{F}\left[z, z^{-1}\right]$ called its symmetric part. There exists an injective algebra homomorphism $\iota: \mathbb{F}[x] \rightarrow \mathbb{F}\left[z, z^{-1}\right]$ that sends $x \mapsto z+z^{-1}$. The image of $\mathbb{F}[x]$ under $\iota$ is the symmetric part of $\mathbb{F}\left[z, z^{-1}\right]$. Via $\iota$ we identify $\mathbb{F}[x]$ with the symmetric part of $\mathbb{F}\left[z, z^{-1}\right]$. So for $n \in \mathbb{N}$ we view

$$
\frac{z^{n+1}-z^{-n-1}}{z-z^{-1}}=z^{n}+z^{n-2}+\cdots+z^{2-n}+z^{-n}
$$

as an element of $\mathbb{F}[x]$.

Lemma 3.3 (see $[24$, p. 326]). For $n \in \mathbb{N}$ we have

$$
U_{n}(x)=\frac{z^{n+1}-z^{-n-1}}{z-z^{-1}},
$$

where we recall $x=z+z^{-1}$.

In this paper, on several occasions we will consider generating functions in an indeterminate $t$. These generating functions involve a formal power series; issues of convergence are not considered. The following generating function will be useful.

Lemma 3.4 (see [23, p. 227]). For an indeterminate $t$,

$$
\sum_{n \in \mathbb{N}} t^{n} U_{n}(x)=\frac{1}{1-t x+t^{2}}
$$

Proof. Using Definition 3.1 one finds that the product $\left(\sum_{n \in \mathbb{N}} t^{n} U_{n}(x)\right)\left(1-t x+t^{2}\right)$ is equal to 1 . Alternatively, use Lemma 3.3. 
The following variations on Lemma 3.4 will be used repeatedly.

Lemma 3.5. For an indeterminate $t$,

$$
\begin{aligned}
& \sum_{n \in \mathbb{N}}(-1)^{n} q^{n} t^{n} U_{n-1}(x)=\frac{-1}{q t+q^{-1} t^{-1}+x}, \\
& \sum_{n \in \mathbb{N}}(-1)^{n} q^{-n} t^{n} U_{n-1}(x)=\frac{-1}{q^{-1} t+q t^{-1}+x} .
\end{aligned}
$$

Lemma 3.6. For an indeterminate $t$,

$$
\sum_{n \in \mathbb{N}}(-1)^{n} t^{n}[n]_{q} U_{n-1}(x)=\frac{t-t^{-1}}{\left(q t+q^{-1} t^{-1}+x\right)\left(q^{-1} t+q t^{-1}+x\right)} .
$$

Proof. Observe that

$$
\begin{aligned}
\sum_{n \in \mathbb{N}}(-1)^{n} t^{n}[n]_{q} U_{n-1}(x) & =\sum_{n \in \mathbb{N}}(-1)^{n} t^{n} \frac{q^{n}-q^{-n}}{q-q^{-1}} U_{n-1}(x) \\
& =\sum_{n \in \mathbb{N}} \frac{(-1)^{n} t^{n} q^{n} U_{n-1}(x)}{q-q^{-1}}-\sum_{n \in \mathbb{N}} \frac{(-1)^{n} t^{n} q^{-n} U_{n-1}(x)}{q-q^{-1}} \\
& =\frac{1}{q-q^{-1}} \frac{-1}{q t+q^{-1} t^{-1}+x}-\frac{1}{q-q^{-1}} \frac{-1}{q^{-1} t+q t^{-1}+x} \\
& =\frac{t-t^{-1}}{\left(q t+q^{-1} t^{-1}+x\right)\left(q^{-1} t+q t^{-1}+x\right)} .
\end{aligned}
$$

\section{Some identities}

In this section we give some identities for later use.

Lemma 4.1. For $r \in \mathbb{Z}$,

$$
[r-1]_{q}-\left(q+q^{-1}\right)[r]_{q}+[r+1]_{q}=0 .
$$

Proof. Use (2.1).

Lemma 4.2. For $r, s \in \mathbb{Z}$ we have

$$
[r-1]_{q}[s-1]_{q}[r-s]_{q}+[r]_{q}[s]_{q}[r-s]_{q}=[r-1]_{q}[s]_{q}[r-s+1]_{q}+[r]_{q}[s-1]_{q}[r-s-1]_{q} .
$$

Proof. Expand each side using (2.1).

Lemma 4.3. For an indeterminate $t$,

$$
\begin{array}{ll}
\sum_{\ell \in \mathbb{N}} t^{2 \ell}=\frac{-t^{-1}}{t-t^{-1}}, & \sum_{\ell \in \mathbb{N}} \ell t^{2 \ell}=\frac{1}{\left(t-t^{-1}\right)^{2}}, \\
\sum_{\ell \in \mathbb{N}} \ell^{2} t^{2 \ell}=-\frac{t+t^{-1}}{\left(t-t^{-1}\right)^{3}}, & \sum_{\ell \in \mathbb{N}}\left(\begin{array}{c}
\ell+1 \\
2
\end{array}\right) t^{2 \ell+1}=\frac{-1}{\left(t-t^{-1}\right)^{3}} .
\end{array}
$$

Proof. These are readily checked. 


\section{The main results}

In this section we express the images (2.8) in the basis (2.16). In what follows, the notation $[u, v]$ means $u v-v u$. We will use a recursion found in [13]; we give a short proof for the sake of completeness.

Lemma 5.1 (see $\left[13\right.$, Section 3.1]). In the algebra $\mathcal{O}_{q}$,

$$
\begin{aligned}
& B_{\alpha_{0}}=A, \quad B_{\delta+\alpha_{0}}=B+\frac{q\left[B_{\delta}, A\right]}{\left(q-q^{-1}\right)\left(q^{2}-q^{-2}\right)}, \\
& B_{n \delta+\alpha_{0}}=B_{(n-2) \delta+\alpha_{0}}+\frac{q\left[B_{\delta}, B_{(n-1) \delta+\alpha_{0}}\right]}{\left(q-q^{-1}\right)\left(q^{2}-q^{-2}\right)}, \quad n \geq 2
\end{aligned}
$$

and also

$$
\begin{aligned}
& B_{\alpha_{1}}=B, \quad B_{\delta+\alpha_{1}}=A-\frac{q\left[B_{\delta}, B\right]}{\left(q-q^{-1}\right)\left(q^{2}-q^{-2}\right)}, \\
& B_{n \delta+\alpha_{1}}=B_{(n-2) \delta+\alpha_{1}}-\frac{q\left[B_{\delta}, B_{(n-1) \delta+\alpha_{1}}\right]}{\left(q-q^{-1}\right)\left(q^{2}-q^{-2}\right)}, \quad n \geq 2 .
\end{aligned}
$$

Proof. We show that

$$
\begin{aligned}
& T_{0}(B)=B+\frac{q\left[B_{\delta}, A\right]}{\left(q-q^{-1}\right)\left(q^{2}-q^{-2}\right)}, \\
& T_{1}^{-1}(A)=A-\frac{q\left[B_{\delta}, B\right]}{\left(q-q^{-1}\right)\left(q^{2}-q^{-2}\right)} .
\end{aligned}
$$

To verify (5.5) (resp. (5.6)) eliminate $B_{\delta}$ using $B_{\delta}=q^{-2} B A-A B$ and compare the result with (2.4) (resp. (2.7)). Lines (5.1), (5.3) follow from (5.5), (5.6) and the construction. Now consider (5.2), (5.4). First assume that $n=2 r+1$ is odd. To verify (5.2), apply $\left(T_{0} T_{1}\right)^{r}$ to each side of (5.5), and use (2.10) along with $T_{1}(B)=B$. To verify (5.4), apply $\left(T_{0} T_{1}\right)^{-r}$ to each side of (5.6), and use (2.10) along with $T_{0}(A)=A$. Next assume that $n=2 r$ is even. To verify (5.2), apply $\left(T_{0} T_{1}\right)^{r}$ to each side of (5.6), and use $(2.10)$ along with $T_{0}(A)=A$, $T_{1}(B)=B$. To verify (5.4), apply $\left(T_{0} T_{1}\right)^{-r}$ to each side of (5.5), and use (2.10) along with $T_{0}(A)=A, T_{1}(B)=B$.

Lemma 5.2. In the algebra $\Delta_{q}$,

$$
B_{\delta}=q^{-1}\left(q^{2}-q^{-2}\right) C-q^{-1}\left(q-q^{-1}\right) \gamma .
$$

Proof. Simplify (2.13) using $q A B-q^{-1} B A=-q B_{\delta}$.

Lemma 5.3. In the algebra $\Delta_{q}$,

$$
\begin{aligned}
& B_{\alpha_{0}}=A, \quad B_{\delta+\alpha_{0}}=B+\frac{[C, A]}{q-q^{-1}}, \\
& B_{n \delta+\alpha_{0}}=B_{(n-2) \delta+\alpha_{0}}+\frac{\left[C, B_{(n-1) \delta+\alpha_{0}}\right]}{q-q^{-1}}, \quad n \geq 2
\end{aligned}
$$

and also

$$
\begin{aligned}
& B_{\alpha_{1}}=B, \quad B_{\delta+\alpha_{1}}=A-\frac{[C, B]}{q-q^{-1}}, \\
& B_{n \delta+\alpha_{1}}=B_{(n-2) \delta+\alpha_{1}}-\frac{\left[C, B_{(n-1) \delta+\alpha_{1}}\right]}{q-q^{-1}}, \quad n \geq 2 .
\end{aligned}
$$


Proof. Evaluate (5.1)-(5.4) using (5.7) and the fact that $\gamma$ is central in $\Delta_{q}$.

Lemma 5.4. In the algebra $\Delta_{q}$,

$$
\begin{aligned}
& \frac{[C, A]}{q-q^{-1}}=-q^{-1} A C-q^{-1}\left(q+q^{-1}\right) B+q^{-1} \beta, \\
& \frac{[C, B]}{q-q^{-1}}=q B C+q\left(q+q^{-1}\right) A-q \alpha .
\end{aligned}
$$

Proof. These equations are a reformulation of (2.11), (2.12).

The following is our first main result.

Theorem 5.5. For $n \geq 0$ the following hold in $\Delta_{q}$ :

$$
\begin{aligned}
B_{n \delta+\alpha_{0}}= & (-1)^{n} q^{-n} A U_{n}(C)+(-1)^{n} q^{-n-1} B U_{n-1}(C)+(-1)^{n} \alpha \sum_{j \in \mathbb{N}} q^{2 j-n+1} U_{n-2 j-2}(C) \\
& +(-1)^{n-1} \beta \sum_{j \in \mathbb{N}} q^{2 j-n} U_{n-2 j-1}(C), \\
B_{n \delta+\alpha_{1}}= & (-1)^{n} q^{n} B U_{n}(C)+(-1)^{n} q^{n+1} A U_{n-1}(C)+(-1)^{n} \beta \sum_{j \in \mathbb{N}} q^{n-2 j-1} U_{n-2 j-2}(C) \\
& +(-1)^{n-1} \alpha \sum_{j \in \mathbb{N}} q^{n-2 j} U_{n-2 j-1}(C) .
\end{aligned}
$$

Proof. By a routine induction on $n$, using Lemmas 5.3, 5.4.

The following is our second main result.

Theorem 5.6. In the algebra $\Delta_{q}$, for $n \geq 1$ the element $B_{n \delta}$ is equal to $(-1)^{n}\left(1-q^{-2}\right)$ times a weighted sum with the following terms and coefficients:

\begin{tabular}{c|c} 
term & coefficient \\
\hline$\Omega$ & $\sum_{\ell \in \mathbb{N}}[n-2 \ell-1]_{q} U_{n-2 \ell-2}(C)$ \\
$\alpha \beta$ & $\sum_{\ell \in \mathbb{N}} \ell^{2}[n-2 \ell]_{q} U_{n-2 \ell-1}(C)$ \\
$\alpha^{2}+\beta^{2}$ & $-\sum_{\ell \in \mathbb{N}}^{(\ell+1} \begin{array}{c}\ell+1 \\
2\end{array}$ \\
$\gamma$ & {$[n]_{q} U_{n-1}(C)+2 \sum_{\ell \in \mathbb{N}}[n-2 \ell-2]_{q} U_{n-2 \ell-2}(C)$} \\
1 & $-[n+1]_{q} U_{n}(C)-[3]_{q}[n-1]_{q} U_{n-2}(C)-[2]_{q}^{2} \sum_{\ell \in \mathbb{N}}[n-2 \ell-3]_{q} U_{n-2 \ell-4}(C)$
\end{tabular}

Proof. We have some preliminary comments. Using (2.12), (2.13),

$$
\begin{aligned}
& B A=q^{2} A B+q\left(q^{2}-q^{-2}\right) C-q\left(q-q^{-1}\right) \gamma, \\
& C A=q^{-2} A C-q^{-1}\left(q^{2}-q^{-2}\right) B+q^{-1}\left(q-q^{-1}\right) \beta, \\
& C A^{2}=q^{-4} A^{2} C-q^{-1}\left(q^{4}-q^{-4}\right) A B+q^{-2}\left(q^{2}-q^{-2}\right) A \beta \\
& \quad-\left(q^{2}-q^{-2}\right)^{2} C+\left(q-q^{-1}\right)\left(q^{2}-q^{-2}\right) \gamma .
\end{aligned}
$$

By [31, Lemma 6.1],

$$
\begin{aligned}
& B A C=q \Omega-q^{3} A^{2}-q^{-1} B^{2}-q^{-1} C^{2}+q^{2} A \alpha+B \beta+C \gamma \\
& C A B=q^{-1} \Omega-q^{-3} A^{2}-q B^{2}-q C^{2}+q^{-2} A \alpha+B \beta+C \gamma .
\end{aligned}
$$


We are done with the preliminary comments. We now define some generating functions in an indeterminate $t$ :

$$
\Phi(t)=\sum_{n=0}^{\infty} t^{n} B_{n \delta+\alpha_{1}}, \quad \Psi(t)=\sum_{n=1}^{\infty} t^{n} B_{n \delta} .
$$

By (2.9),

$$
\Psi(t)=q^{-2} t \Phi(t) A-t A \Phi(t)+\left(q^{-2}-1\right) t^{2}(\Phi(t))^{2} .
$$

By (5.10), (5.11),

$$
\frac{[C, \Phi(t)]}{q-q^{-1}}=A+t^{-1} B+\left(t-t^{-1}\right) \Phi(t) .
$$

We next consider what the second equation in Theorem 5.5 implies about $\Phi(t)$. Using Lemma 3.4,

$$
\sum_{n \in \mathbb{N}}(-1)^{n} q^{n} t^{n} U_{n}(x)=\frac{q^{-1} t^{-1}}{q t+q^{-1} t^{-1}+x} .
$$

Using Lemma 3.5,

$$
\sum_{n \in \mathbb{N}}(-1)^{n} q^{n+1} t^{n} U_{n-1}(x)=\frac{-q}{q t+q^{-1} t^{-1}+x} .
$$

We have

$$
\begin{aligned}
\sum_{n \in \mathbb{N}}( & -1)^{n} t^{n} \sum_{j \in \mathbb{N}} q^{n-2 j-1} U_{n-2 j-2}(x) \\
& =\sum_{n \in \mathbb{N}} \sum_{j \in \mathbb{N}}(-1)^{n} t^{n} q^{n-2 j-1} U_{n-2 j-2}(x) \\
& =-\sum_{n \in \mathbb{N}} \sum_{j \in \mathbb{N}}(-1)^{n-2 j-1} t^{n-2 j-1} q^{n-2 j-1} U_{n-2 j-2}(x) t^{2 j+1} \\
& \left.=-\sum_{N \in \mathbb{N}} \sum_{j \in \mathbb{N}}(-1)^{N} t^{N} q^{N} U_{N-1}(x) t^{2 j+1} \quad \text { (change var. } N=n-2 j-1\right) \\
& =-\left(\sum_{N \in \mathbb{N}}(-1)^{N} t^{N} q^{N} U_{N-1}(x)\right)\left(\sum_{j \in \mathbb{N}} t^{2 j+1}\right) \\
& =\frac{-1}{q t+q^{-1} t^{-1}+x} \frac{1}{t-t^{-1}} \\
& =\frac{-1}{\left(t-t^{-1}\right)\left(q t+q^{-1} t^{-1}+x\right)} .
\end{aligned}
$$

Similarly,

$$
\sum_{n \in \mathbb{N}}(-1)^{n-1} t^{n} \sum_{j \in \mathbb{N}} q^{n-2 j} U_{n-2 j-1}(x)=\frac{-t^{-1}}{\left(t-t^{-1}\right)\left(q t+q^{-1} t^{-1}+x\right)} .
$$

By these comments and the second equation in Theorem 5.5,

$$
\Phi(t)\left(q t+q^{-1} t^{-1}+C\right)=q^{-1} t^{-1} B-q A-\frac{\beta}{t-t^{-1}}-\frac{t^{-1} \alpha}{t-t^{-1}} .
$$


By (5.16) and (5.17),

$$
\left(q^{-1} t+q t^{-1}+C\right) \Phi(t)=q t^{-1} B-q^{-1} A-\frac{\beta}{t-t^{-1}}-\frac{t^{-1} \alpha}{t-t^{-1}} .
$$

In (5.15), we multiply each side on the left by $q^{-1} t+q t^{-1}+C$ and on the right by $q t+q^{-1} t^{-1}+C$. We evaluate the result using (5.17), (5.18) to obtain

$$
\begin{aligned}
\left(q^{-1} t+\right. & \left.q t^{-1}+C\right) \Psi(t)\left(q t+q^{-1} t^{-1}+C\right) \\
= & q^{-2} t\left(q t^{-1} B-q^{-1} A-\frac{\beta}{t-t^{-1}}-\frac{t^{-1} \alpha}{t-t^{-1}}\right) A\left(q t+q^{-1} t^{-1}+C\right) \\
& -t\left(q^{-1} t+q t^{-1}+C\right) A\left(q^{-1} t^{-1} B-q A-\frac{\beta}{t-t^{-1}}-\frac{t^{-1} \alpha}{t-t^{-1}}\right) \\
& +\left(q^{-2}-1\right) t^{2}\left(q t^{-1} B-q^{-1} A-\frac{\beta}{t-t^{-1}}-\frac{t^{-1} \alpha}{t-t^{-1}}\right) \\
& \times\left(q^{-1} t^{-1} B-q A-\frac{\beta}{t-t^{-1}}-\frac{t^{-1} \alpha}{t-t^{-1}}\right) .
\end{aligned}
$$

Evaluating the above equation using the preliminary comments, we find that

$$
\left(q^{-1} t+q t^{-1}+C\right) \Psi(t)\left(q t+q^{-1} t^{-1}+C\right)
$$

is equal to $1-q^{-2}$ times

$$
\Omega-\frac{\left(t+t^{-1}\right) \alpha \beta}{\left(t-t^{-1}\right)^{2}}-\frac{\alpha^{2}+\beta^{2}}{\left(t-t^{-1}\right)^{2}}-\left(t+t^{-1}\right) \gamma+\left(q+q^{-1}\right)\left(t+t^{-1}\right) C+C^{2} .
$$

Consequently $\Psi(t)$ is equal to $1-q^{-2}$ times

$$
F_{1}(t, C) \Omega+F_{2}(t, C) \alpha \beta+F_{3}(t, C)\left(\alpha^{2}+\beta^{2}\right)+F_{4}(t, C) \gamma+F_{5}(t, C),
$$

where

$$
\begin{aligned}
& F_{1}(t, x)=\frac{1}{\left(q t+q^{-1} t^{-1}+x\right)\left(q^{-1} t+q t^{-1}+x\right)}, \\
& F_{2}(t, x)=-\frac{t+t^{-1}}{\left(t-t^{-1}\right)^{2}\left(q t+q^{-1} t^{-1}+x\right)\left(q^{-1} t+q t^{-1}+x\right)}, \\
& F_{3}(t, x)=\frac{-1}{\left(t-t^{-1}\right)^{2}\left(q t+q^{-1} t^{-1}+x\right)\left(q^{-1} t+q t^{-1}+x\right)}, \\
& F_{4}(t, x)=-\frac{t+t^{-1}}{\left(q t+q^{-1} t^{-1}+x\right)\left(q^{-1} t+q t^{-1}+x\right)}, \\
& F_{5}(t, x)=\frac{\left(q+q^{-1}\right)\left(t+t^{-1}\right) x+x^{2}}{\left(q t+q^{-1} t^{-1}+x\right)\left(q^{-1} t+q t^{-1}+x\right)} .
\end{aligned}
$$

We now compare the $\left\{F_{i}\right\}_{i=1}^{5}$ with the coefficients shown in the table of the theorem statement.

Concerning $F_{1}$,

$$
\begin{aligned}
\sum_{n=1}^{\infty}(-1)^{n} t^{n} \sum_{\ell \in \mathbb{N}}[n-2 \ell-1]_{q} U_{n-2 \ell-2}(x) \\
=\sum_{n=1}^{\infty} \sum_{\ell \in \mathbb{N}}(-1)^{n} t^{n}[n-2 \ell-1]_{q} U_{n-2 \ell-2}(x)
\end{aligned}
$$




$$
\begin{aligned}
& =-\sum_{n=1}^{\infty} \sum_{\ell \in \mathbb{N}}(-1)^{n-2 \ell-1} t^{n-2 \ell-1}[n-2 \ell-1]_{q} U_{n-2 \ell-2}(x) t^{2 \ell+1} \\
& \left.=-\sum_{N \in \mathbb{N}} \sum_{\ell \in \mathbb{N}}(-1)^{N} t^{N}[N]_{q} U_{N-1}(x) t^{2 \ell+1} \quad \text { (change var. } N=n-2 \ell-1\right) \\
& =-\left(\sum_{N \in \mathbb{N}}(-1)^{N} t^{N}[N]_{q} U_{N-1}(x)\right)\left(\sum_{\ell \in \mathbb{N}} t^{2 \ell+1}\right) \\
& =\frac{t-t^{-1}}{\left(q t+q^{-1} t^{-1}+x\right)\left(q^{-1} t+q t^{-1}+x\right)} \frac{1}{t-t^{-1}} \\
& =\frac{1}{\left(q t+q^{-1} t^{-1}+x\right)\left(q^{-1} t+q t^{-1}+x\right)} \\
& =F_{1}(t, x) .
\end{aligned}
$$

Concerning $F_{2}$,

$$
\begin{aligned}
\sum_{n=1}^{\infty}( & -1)^{n} t^{n} \sum_{\ell \in \mathbb{N}} \ell^{2}[n-2 \ell]_{q} U_{n-2 \ell-1}(x) \\
& =\sum_{n=1}^{\infty} \sum_{\ell \in \mathbb{N}}(-1)^{n} t^{n}[n-2 \ell]_{q} U_{n-2 \ell-1}(x) \ell^{2} \\
& =\sum_{n=1}^{\infty} \sum_{\ell \in \mathbb{N}}(-1)^{n-2 \ell} t^{n-2 \ell}[n-2 \ell]_{q} U_{n-2 \ell-1}(x) \ell^{2} t^{2 \ell} \\
& =\sum_{N \in \mathbb{N}} \sum_{\ell \in \mathbb{N}}(-1)^{N} t^{N}[N]_{q} U_{N-1}(x) \ell^{2} t^{2 \ell} \quad(\text { change var. } N=n-2 \ell) \\
& =\left(\sum_{N \in \mathbb{N}}(-1)^{N} t^{N}[N]_{q} U_{N-1}(x)\right)\left(\sum_{\ell \in \mathbb{N}} \ell^{2} t^{2 \ell}\right) \\
& =-\frac{t-t^{-1}}{\left(q t+q^{-1} t^{-1}+x\right)\left(q^{-1} t+q t^{-1}+x\right)} \frac{t+t^{-1}}{\left(t-t^{-1}\right)^{3}} \\
& =-\frac{t+t^{-1}}{\left(t-t^{-1}\right)^{2}\left(q t+q^{-1} t^{-1}+x\right)\left(q^{-1} t+q t^{-1}+x\right)} \\
& =F_{2}(t, x) .
\end{aligned}
$$

Concerning $F_{3}$,

$$
\begin{aligned}
-\sum_{n=1}^{\infty} & (-1)^{n} t^{n} \sum_{\ell \in \mathbb{N}}\left(\begin{array}{c}
\ell+1 \\
2
\end{array}\right)[n-2 \ell-1]_{q} U_{n-2 \ell-2}(x) \\
& =-\sum_{n=1}^{\infty} \sum_{\ell \in \mathbb{N}}(-1)^{n} t^{n}[n-2 \ell-1]_{q} U_{n-2 \ell-2}(x)\left(\begin{array}{c}
\ell+1 \\
2
\end{array}\right) \\
& =\sum_{n=1}^{\infty} \sum_{\ell \in \mathbb{N}}(-1)^{n-2 \ell-1} t^{n-2 \ell-1}[n-2 \ell-1]_{q} U_{n-2 \ell-2}(x)\left(\begin{array}{c}
\ell+1 \\
2
\end{array}\right) t^{2 \ell+1} \\
& \left.=\sum_{N \in \mathbb{N}} \sum_{\ell \in \mathbb{N}}(-1)^{N} t^{N}[N]_{q} U_{N-1}(x)\left(\begin{array}{c}
\ell+1 \\
2
\end{array}\right) t^{2 \ell+1} \quad \text { change var. } N=n-2 \ell-1\right) \\
& =\left(\sum_{N \in \mathbb{N}}(-1)^{N} t^{N}[N]_{q} U_{N-1}(x)\right)\left(\sum_{\ell \in \mathbb{N}}\left(\begin{array}{c}
\ell+1 \\
2
\end{array}\right) t^{2 \ell+1}\right)
\end{aligned}
$$




$$
\begin{aligned}
& =\frac{t-t^{-1}}{\left(q t+q^{-1} t^{-1}+x\right)\left(q^{-1} t+q t^{-1}+x\right)} \frac{-1}{\left(t-t^{-1}\right)^{3}} \\
& =\frac{-1}{\left(t-t^{-1}\right)^{2}\left(q t+q^{-1} t^{-1}+x\right)\left(q^{-1} t+q t^{-1}+x\right)} \\
& =F_{3}(t, x) .
\end{aligned}
$$

Concerning $F_{4}$,

$$
\begin{aligned}
\sum_{n=1}^{\infty}(-1)^{n} t^{n}[n]_{q} U_{n-1}(x) & \\
\quad= & \sum_{n \in \mathbb{N}}(-1)^{n} t^{n}[n]_{q} U_{n-1}(x) \\
& =\frac{t-t^{-1}}{\left(q t+q^{-1} t^{-1}+x\right)\left(q^{-1} t+q t^{-1}+x\right)}
\end{aligned}
$$

and also

$$
\begin{aligned}
\sum_{n=1}^{\infty}( & -1)^{n} t^{n} \sum_{\ell \in \mathbb{N}}[n-2 \ell-2]_{q} U_{n-2 \ell-3}(x) \\
& =\sum_{n=1}^{\infty} \sum_{\ell \in \mathbb{N}}(-1)^{n} t^{n}[n-2 \ell-2]_{q} U_{n-2 \ell-3}(x) \\
& =\sum_{n=1}^{\infty} \sum_{\ell \in \mathbb{N}}(-1)^{n-2 \ell-2} t^{n-2 \ell-2}[n-2 \ell-2]_{q} U_{n-2 \ell-3}(x) t^{2 \ell+2} \\
& =\sum_{N \in \mathbb{N}} \sum_{\ell \in \mathbb{N}}(-1)^{N} t^{N}[N]_{q} U_{N-1}(x) t^{2 \ell+2} \quad(\text { change var. } N=n-2 \ell-2) \\
& =\left(\sum_{N \in \mathbb{N}}(-1)^{N} t^{N}[N]_{q} U_{N-1}(x)\right)\left(\sum_{\ell \in \mathbb{N}} t^{2 \ell+2}\right) \\
& =-\frac{t-t^{-1}}{\left(q t+q^{-1} t^{-1}+x\right)\left(q^{-1} t+q t^{-1}+x\right)} \frac{t}{t-t^{-1}} \\
& =-\frac{t}{\left(q t+q^{-1} t^{-1}+x\right)\left(q^{-1} t+q t^{-1}+x\right)} .
\end{aligned}
$$

Note that (5.20) plus twice (5.21) is equal to $F_{4}(t, x)$.

Concerning $F_{5}$,

$$
\begin{aligned}
\sum_{n=1}^{\infty}( & -1)^{n} t^{n}[n+1]_{q} U_{n}(x) \\
& =-1+\sum_{n \in \mathbb{N}}(-1)^{n} t^{n}[n+1]_{q} U_{n}(x) \\
& =-1-t^{-1} \sum_{n \in \mathbb{N}}(-1)^{n+1} t^{n+1}[n+1]_{q} U_{n}(x) \\
& =-1-t^{-1} \sum_{N \in \mathbb{N}}(-1)^{N} t^{N}[N]_{q} U_{N-1}(x) \quad(\text { change var. } N=n+1) \\
& =-1-\frac{t^{-1}\left(t-t^{-1}\right)}{\left(q t+q^{-1} t^{-1}+x\right)\left(q^{-1} t+q t^{-1}+x\right)}
\end{aligned}
$$


and also

$$
\begin{aligned}
& \sum_{n=1}^{\infty}(-1)^{n} t^{n}[n-1]_{q} U_{n-2}(x) \\
& \quad=-t \sum_{n=1}^{\infty}(-1)^{n-1} t^{n-1}[n-1]_{q} U_{n-2}(x) \\
& \left.\quad=-t \sum_{N \in \mathbb{N}}(-1)^{N} t^{N}[N]_{q} U_{N-1}(x) \quad \text { (change var. } N=n-1\right) \\
& \quad=-\frac{t\left(t-t^{-1}\right)}{\left(q t+q^{-1} t^{-1}+x\right)\left(q^{-1} t+q t^{-1}+x\right)},
\end{aligned}
$$

and also

$$
\begin{aligned}
\sum_{n=1}^{\infty}( & -1)^{n} t^{n} \sum_{\ell \in \mathbb{N}}[n-2 \ell-3]_{q} U_{n-2 \ell-4}(x) \\
& =\sum_{n=1}^{\infty} \sum_{\ell \in \mathbb{N}}(-1)^{n} t^{n}[n-2 \ell-3]_{q} U_{n-2 \ell-4}(x) \\
& =-\sum_{n=1}^{\infty} \sum_{\ell \in \mathbb{N}}(-1)^{n-2 \ell-3} t^{n-2 \ell-3}[n-2 \ell-3]_{q} U_{n-2 \ell-4}(x) t^{2 \ell+3} \\
& =-\sum_{N \in \mathbb{N}} \sum_{\ell \in \mathbb{N}}(-1)^{N} t^{N}[N]_{q} U_{N-1}(x) t^{2 \ell+3} \quad(\text { change var. } N=n-2 \ell-3) \\
& =-\left(\sum_{N \in \mathbb{N}}(-1)^{N} t^{N}[N]_{q} U_{N-1}(x)\right)\left(\sum_{\ell \in \mathbb{N}} t^{2 \ell+3}\right) \\
& =\frac{t-t^{-1}}{\left(q t+q^{-1} t^{-1}+x\right)\left(q^{-1} t+q t^{-1}+x\right)} \frac{t^{2}}{t-t^{-1}} \\
& =\frac{t^{2}}{\left(q t+q^{-1} t^{-1}+x\right)\left(q^{-1} t+q t^{-1}+x\right)} .
\end{aligned}
$$

Note that $(-1)$ times $(5.22)$ minus $[3]_{q}$ times $(5.23)$ minus $[2]_{q}^{2}$ times $(5.24)$ is equal to $F_{5}(t, x)$. The result follows from the above comments.

Recall the center $Z\left(\Delta_{q}\right)$.

Corollary 5.7. For $n \geq 1$ the element $B_{n \delta}$ is contained in the subalgebra of $\Delta_{q}$ generated by $C$ and $Z\left(\Delta_{q}\right)$.

We finish the paper with some comments.

Here is another version of Theorem 5.5.

Proposition 5.8. For $n \geq 0$ the following hold in $\Delta_{q}$ :

$$
\begin{aligned}
B_{n \delta+\alpha_{0}}= & (-1)^{n} q^{n} U_{n}(C) A+(-1)^{n} q^{n+1} U_{n-1}(C) B+(-1)^{n} \alpha \sum_{j \in \mathbb{N}} q^{n-2 j-1} U_{n-2 j-2}(C) \\
& +(-1)^{n-1} \beta \sum_{j \in \mathbb{N}} q^{n-2 j} U_{n-2 j-1}(C), \\
B_{n \delta+\alpha_{1}}= & (-1)^{n} q^{-n} U_{n}(C) B+(-1)^{n} q^{-n-1} U_{n-1}(C) A+(-1)^{n} \beta \sum_{j \in \mathbb{N}} q^{2 j-n+1} U_{n-2 j-2}(C) \\
& +(-1)^{n-1} \alpha \sum_{j \in \mathbb{N}} q^{2 j-n} U_{n-2 j-1}(C) .
\end{aligned}
$$


Proof. Similar to the proof of Theorem 5.5.

The following result might be of independent interest.

Proposition 5.9. For $n \geq 1$ the following holds in $\Delta_{q}$ :

$$
\begin{aligned}
U_{n}(C) A= & q^{-2 n} A U_{n}(C)-q^{2}\left(q-q^{-1}\right) A \sum_{\ell \in \mathbb{N}}[2 n-4 \ell-2]_{q} U_{n-2 \ell-2}(C) \\
& -q^{-1}\left(q-q^{-1}\right) B \sum_{\ell \in \mathbb{N}}[2 n-4 \ell]_{q} U_{n-2 \ell-1}(C) \\
& +\left(q-q^{-1}\right)^{2} \alpha \sum_{\ell \in \mathbb{N}}[n-2 \ell-1]_{q}[\ell+1]_{q}[n-\ell]_{q} U_{n-2 \ell-2}(C) \\
& +\left(q-q^{-1}\right) \beta \sum_{\ell \in \mathbb{N}}[n-2 \ell]_{q}\left(q^{\ell-n}[\ell+1]_{q}-q^{n-\ell+1}[\ell]_{q}\right) U_{n-2 \ell-1}(C)
\end{aligned}
$$

and also

$$
\begin{aligned}
U_{n}(C) B= & q^{2 n} B U_{n}(C)+q^{-2}\left(q-q^{-1}\right) B \sum_{\ell \in \mathbb{N}}[2 n-4 \ell-2]_{q} U_{n-2 \ell-2}(C) \\
& +q\left(q-q^{-1}\right) A \sum_{\ell \in \mathbb{N}}[2 n-4 \ell]_{q} U_{n-2 \ell-1}(C) \\
& +\left(q-q^{-1}\right)^{2} \beta \sum_{\ell \in \mathbb{N}}[n-2 \ell-1]_{q}[\ell+1]_{q}[n-\ell]_{q} U_{n-2 \ell-2}(C) \\
& -\left(q-q^{-1}\right) \alpha \sum_{\ell \in \mathbb{N}}[n-2 \ell]_{q}\left(q^{n-\ell}[\ell+1]_{q}-q^{\ell-n-1}[\ell]_{q}\right) U_{n-2 \ell-1}(C) .
\end{aligned}
$$

Proof. We use induction on $n$. For $n=1$ the equations in the proposition statement are reformulations of (2.11), (2.12). For $n \geq 2$ we proceed as follows. To obtain the first (resp. second) equation in the proposition statement, multiply each side of (2.12) (resp. (2.11)) on the left by $U_{n-1}(C)$, and evaluate the result using $C U_{n-1}(C)=U_{n}(C)+U_{n-2}(C)$ along with induction and Lemmas 4.1, 4.2.

In the algebra $\mathcal{O}_{q}$ the elements $\left\{B_{n \delta}\right\}_{n=1}^{\infty}$ are defined using the formula (2.9). This formula is not symmetric in $\alpha_{0}, \alpha_{1}$. As shown in [13], there is another formula for $\left\{B_{n \delta}\right\}_{n=1}^{\infty}$ that interchanges the roles of $\alpha_{0}, \alpha_{1}$. According to [13, Section 5.2] the following holds in $\mathcal{O}_{q}$ for $n \geq 1$ :

$$
B_{n \delta}=q^{-2} B B_{(n-1) \delta+\alpha_{0}}-B_{(n-1) \delta+\alpha_{0}} B+\left(q^{-2}-1\right) \sum_{\ell=0}^{n-2} B_{\ell \delta+\alpha_{0}} B_{(n-\ell-2) \delta+\alpha_{0}} .
$$

We now sketch a proof of Theorem 5.6 that uses (5.25) instead of (2.9). Following (5.14), for the algebra $\Delta_{q}$ we define

$$
\tilde{\Phi}(t)=\sum_{n=0}^{\infty} t^{n} B_{n \delta+\alpha_{0}} .
$$

By (5.14), (5.25), (5.26) we obtain

$$
\Psi(t)=q^{-2} t B \tilde{\Phi}(t)-t \tilde{\Phi}(t) B+\left(q^{-2}-1\right) t^{2}(\tilde{\Phi}(t))^{2} .
$$

By (5.8), (5.9),

$$
\frac{[\tilde{\Phi}(t), C]}{q-q^{-1}}=t^{-1} A+B+\left(t-t^{-1}\right) \tilde{\Phi}(t) .
$$


From the first equation in Theorem 5.5 we obtain

$$
\tilde{\Phi}(t)\left(q^{-1} t+q t^{-1}+C\right)=q t^{-1} A-q^{-1} B-\frac{\alpha}{t-t^{-1}}-\frac{t^{-1} \beta}{t-t^{-1}} .
$$

By (5.28) and (5.29),

$$
\left(q t+q^{-1} t^{-1}+C\right) \tilde{\Phi}(t)=q^{-1} t^{-1} A-q B-\frac{\alpha}{t-t^{-1}}-\frac{t^{-1} \beta}{t-t^{-1}} .
$$

In (5.27), we multiply each side on the left by $q t+q^{-1} t^{-1}+C$ and on the right by $q^{-1} t+q t^{-1}+C$. We evaluate the result using (5.29), (5.30) to obtain

$$
\begin{aligned}
(q t+ & \left.q^{-1} t^{-1}+C\right) \Psi(t)\left(q^{-1} t+q t^{-1}+C\right) \\
= & q^{-2} t\left(q t+q^{-1} t^{-1}+C\right) B\left(q t^{-1} A-q^{-1} B-\frac{\alpha}{t-t^{-1}}-\frac{t^{-1} \beta}{t-t^{-1}}\right) \\
& -t\left(q^{-1} t^{-1} A-q B-\frac{\alpha}{t-t^{-1}}-\frac{t^{-1} \beta}{t-t^{-1}}\right) B\left(q^{-1} t+q t^{-1}+C\right) \\
& +\left(q^{-2}-1\right) t^{2}\left(q^{-1} t^{-1} A-q B-\frac{\alpha}{t-t^{-1}}-\frac{t^{-1} \beta}{t-t^{-1}}\right) \\
& \times\left(q t^{-1} A-q^{-1} B-\frac{\alpha}{t-t^{-1}}-\frac{t^{-1} \beta}{t-t^{-1}}\right) .
\end{aligned}
$$

Evaluating this equation using

$$
\begin{aligned}
& B A=q^{2} A B+q\left(q^{2}-q^{-2}\right) C-q\left(q-q^{-1}\right) \gamma, \\
& C B=q^{2} B C+q\left(q^{2}-q^{-2}\right) A-q\left(q-q^{-1}\right) \alpha, \\
& C B^{2}=q^{4} B^{2} C+q^{3}\left(q^{4}-q^{-4}\right) A B-q^{2}\left(q^{2}-q^{-2}\right) B \alpha \\
& \quad+q^{4}\left(q^{2}-q^{-2}\right)^{2} C-q^{4}\left(q-q^{-1}\right)\left(q^{2}-q^{-2}\right) \gamma
\end{aligned}
$$

and

$$
\begin{aligned}
& A B C=q^{-1} \Omega-q A^{2}-q^{-3} B^{2}-q C^{2}+A \alpha+q^{-2} B \beta+C \gamma, \\
& C B A=q \Omega-q^{-1} A^{2}-q^{3} B^{2}-q^{-1} C^{2}+A \alpha+q^{2} B \beta+C \gamma
\end{aligned}
$$

we find that

$$
\left(q t+q^{-1} t^{-1}+C\right) \Psi(t)\left(q^{-1} t+q t^{-1}+C\right)
$$

is equal to $1-q^{-2}$ times

$$
\Omega-\frac{\left(t+t^{-1}\right) \alpha \beta}{\left(t-t^{-1}\right)^{2}}-\frac{\alpha^{2}+\beta^{2}}{\left(t-t^{-1}\right)^{2}}-\left(t+t^{-1}\right) \gamma+\left(q+q^{-1}\right)\left(t+t^{-1}\right) C+C^{2} .
$$

After this point, the present proof is the same as the original proof.

\section{Acknowledgements}

The author thanks Pascal Baseilhac and Samuel Belliard for giving this paper a close reading and offering valuable suggestions. 


\section{References}

[1] Alperin R.C., Notes: $\operatorname{PSL}_{2}(Z)=\mathbb{Z}_{2} \star \mathbb{Z}_{3}$, Amer. Math. Monthly 100 (1993), 385-386.

[2] Alperin R.C., The modular tree of Pythagoras, Amer. Math. Monthly 112 (2005), 807-816, math.HO/0010281.

[3] Baseilhac P., An integrable structure related with tridiagonal algebras, Nuclear Phys. B 705 (2005), 605619, math-ph/0408025.

[4] Baseilhac P., Deformed Dolan-Grady relations in quantum integrable models, Nuclear Phys. B 709 (2005), 491-521, hep-th/0404149.

[5] Baseilhac P., A family of tridiagonal pairs and related symmetric functions, J. Phys. A: Math. Gen. 39 (2006), 11773-11791, math-ph/0604035.

[6] Baseilhac P., The $q$-deformed analogue of the Onsager algebra: beyond the Bethe ansatz approach, Nuclear Phys. B 754 (2006), 309-328, math-ph/0604036.

[7] Baseilhac P., Belliard S., An attractive basis for the $q$-Onsager algebra, arXiv:1704.02950.

[8] Baseilhac P., Belliard S., Generalized q-Onsager algebras and boundary affine Toda field theories, Lett. Math. Phys. 93 (2010), 213-228, arXiv:0906.1215.

[9] Baseilhac P., Belliard S., The half-infinite $X X Z$ chain in Onsager's approach, Nuclear Phys. B 873 (2013), 550-584, arXiv:1211.6304.

[10] Baseilhac P., Koizumi K., A deformed analogue of Onsager's symmetry in the $X X Z$ open spin chain, J. Stat. Mech. Theory Exp. 2005 (2005), P10005, 15 pages, hep-th/0507053.

[11] Baseilhac P., Koizumi K., A new (in)finite-dimensional algebra for quantum integrable models, Nuclear Phys. B $\mathbf{7 2 0}$ (2005), 325-347, math-ph/0503036.

[12] Baseilhac P., Koizumi K., Exact spectrum of the $X X Z$ open spin chain from the $q$-Onsager algebra representation theory, J. Stat. Mech. Theory Exp. 2007 (2007), P09006, 27 pages, hep-th/0703106.

[13] Baseilhac P., Kolb S., Braid group action and root vectors for the $q$-Onsager algebra, arXiv:1706.08747.

[14] Baseilhac P., Shigechi K., A new current algebra and the reflection equation, Lett. Math. Phys. 92 (2010), 47-65, arXiv:0906.1482.

[15] Baseilhac P., Vu T.T., Analogues of Lusztig's higher order relations for the q-Onsager algebra, J. Math. Phys. 55 (2014), 081707, 21 pages, arXiv:1312.3433.

[16] Damiani I., A basis of type Poincaré-Birkhoff-Witt for the quantum algebra of sl(2), J. Algebra 161 (1993), 291-310.

[17] Dolan L., Grady M., Conserved charges from self-duality, Phys. Rev. D 25 (1982), 1587-1604.

[18] Huang H.-W., Finite-dimensional irreducible modules of the universal Askey-Wilson algebra, Comm. Math. Phys. 340 (2015), 959-984, arXiv:1210.1740.

[19] Ito T., Nomura K., Terwilliger P., A classification of sharp tridiagonal pairs, Linear Algebra Appl. 435 (2011), 1857-1884, arXiv:1001.1812.

[20] Ito T., Tanabe K., Terwilliger P., Some algebra related to $P$ - and $Q$-polynomial association schemes, in Codes and Association Schemes (Piscataway, NJ, 1999), DIMACS Ser. Discrete Math. Theoret. Comput. Sci., Vol. 56, Amer. Math. Soc., Providence, RI, 2001, 167-192, math.CO/0406556.

[21] Ito T., Terwilliger P., Tridiagonal pairs of q-Racah type, J. Algebra 322 (2009), 68-93, arXiv:0807.0271.

[22] Ito T., Terwilliger P., The augmented tridiagonal algebra, Kyushu J. Math. 64 (2010), 81-144, arXiv:0807.3990.

[23] Koekoek R., Lesky P.A., Swarttouw R.F., Hypergeometric orthogonal polynomials and their $q$-analogues, Springer Monographs in Mathematics, Springer-Verlag, Berlin, 2010.

[24] Mason J.C., Handscomb D.C., Chebyshev polynomials, Chapman \& Hall/CRC, Boca Raton, FL, 2003.

[25] Onsager L., Crystal statistics. I. A two-dimensional model with an order-disorder transition, Phys. Rev. 65 (1944), 117-149.

[26] Perk J.H.H., Star-triangle equations, quantum Lax pairs, and higher genus curves, in Theta Functions Bowdoin 1987, Part 1 (Brunswick, ME, 1987), Proc. Sympos. Pure Math., Vol. 49, Amer. Math. Soc., Providence, RI, 1989, 341-354.

[27] Terwilliger P., The subconstituent algebra of an association scheme. III, J. Algebraic Combin. 2 (1993), $177-210$. 
[28] Terwilliger P., Two linear transformations each tridiagonal with respect to an eigenbasis of the other, Linear Algebra Appl. 330 (2001), 149-203, math.RA/0406555.

[29] Terwilliger P., Two relations that generalize the $q$-Serre relations and the Dolan-Grady relations, in Physics and Combinatorics 1999 (Nagoya), World Sci. Publ., River Edge, NJ, 2001, 377-398, math.QA/0307016.

[30] Terwilliger P., An algebraic approach to the Askey scheme of orthogonal polynomials, in Orthogonal Polynomials and Special Functions, Lecture Notes in Math., Vol. 1883, Springer, Berlin, 2006, 255-330, math.QA/0408390.

[31] Terwilliger P., The universal Askey-Wilson algebra, SIGMA 7 (2011), 069, 24 pages, arXiv:1104.2813.

[32] Terwilliger P., The $q$-Onsager algebra and the positive part of $U_{q}\left(\widehat{\mathfrak{s l}}_{2}\right)$, Linear Algebra Appl. 521 (2017), 19-56, arXiv:1506.08666.

[33] Terwilliger P., Vidunas R., Leonard pairs and the Askey-Wilson relations, J. Algebra Appl. 3 (2004), 411426, math.QA/0305356.

[34] Zhedanov A.S., "Hidden symmetry" of Askey-Wilson polynomials, Theoret. and Math. Phys. 89 (1991), $1146-1157$. 